\title{
Propuesta de implementación de la metodología Inbound Marketing (marketing de contenidos) para mejorar las acciones de mercadeo de la Cooperativa multiactiva "Copservir Ltda"
}

\author{
Sandra Rocio Mondragon A. ${ }^{1}$ \\ Martha Lucía Fuertes D. ${ }^{2}$ \\ Julio Cesar Montoya R. ${ }^{3}$
}

\section{RESUMEN}

El presente documento, utiliza la metodología "marketing de contenidos (Inbound Marketing), que consiste en una serie de técnicas de marketing dirigidas a incrementar el número de visitantes virtuales de una página web, un blog o un perfil en redes sociales, para que se conviertan en leads (registros o cupones con los datos de aquellas personas potencialmente interesadas en la empresa).

La primera fase del uso de esta metodología, corresponde al diagnóstico de las acciones de marketing que desarrolla la organización a través de interacción virtual con los clientes.

La segunda fase concierne a la identificación de los elementos estructurales de esta metodología, requeridos para su cabal aplicación y el grado de cumplimiento de dichos elementos por parte de Copservir Ltda; es decir si ya existían, si ya se aplican, si vale la pena implementarlos, etc.

En la parte final del documento, se plantean las propuestas de implementación del Marketing de contenidos en la Cooperativa Multiactiva Copservir Ltda, más precisamente en lo que compete a su tienda virtual y su dinámica en las redes sociales.

Palabras clave: acciones de marketing, leads, blog, promoción, estrategia

\footnotetext{
${ }_{1}^{1}$ Administradora de Empresas, Especialista en Alta Gerencia, Magister en Dirección y Administración de empresas. Decana Nacional de la Escuela de Ciencias Administrativas, Contables, Económicas y de Negocios - ECACEN de la Universidad Nacional Abierta y a Distancia -UNAD. sandra.mondragon@unad.edu.co

${ }^{2}$ Contadora Pública, Especialista en Finanzas, Maestría en Administración, docente Universidad Nacional Abierta y a Distancia - UNAD. martha.fuertes@unad.edu.co

${ }^{3}$ Administrador de Empresas, Especialista en Pedagogía para el Desarrollo del Aprendizaje Autónomo, Maestría en Administración de Empresas.Docente de la Universidad Nacional Abierta y a Distancia UNAD. julio.montoya@unad.edu.co
} 


\section{Introducción}

En tiempos de globalización, de internacionalización de mercados, de inmediatez informática, y de virtualidad extrema es ampliamente aceptado que tanto las organizaciones empresariales como los individuos vean el Internet como su mayor fuente de beneficios.

De hecho, contar con una tienda virtual, un blog, o tener un espacio "en línea" ofrece un potencial casi infinito para las compañías en su propósito puntual de obtener el mayor número de ventajas competitivas que le permitan marcar una diferencia con sus pares, dicho de otra manera, estar en línea las 24 horas del día, es estar potencialmente abierto al advenimiento de miles de negocios.

La comercialización que el Internet posibilita a sus usuarios, es tan eficaz que el volumen de oportunidades no tiene precedentes en la historia, , lo que habría sido una utopía en décadas anteriores, debido a la enorme cantidad de recursos necesarios que involucra dicho proceso (Simpson \& Jevons, 2009).

Precisamente, el objeto de esta investigación, es aplicar una metodología que permita mejorar las acciones de mercadeo de la Cooperativa Multiactiva Copservir Ltda, por medio de la estrategia de Inbound Marketing, focalizada en incrementar el número de visitantes que registra la tienda virtual con el fin de que estos, acaben convirtiéndose en leads (es decir, registros o cupones con los datos de aquellas personas que se han interesado por los contenidos online de la empresa), terminen convirtiéndose en clientes asiduos de la organización.

\section{Marco Teórico}

\section{Inbound Marketing (Definiciones)}

En lo que hace referencia a esta nueva estrategia de Mercado de índole virtual, la autora Wilcok, (2011), en el documento publicado como Marketing de Contenidos. Crear para convertir, explica: El concepto de inbound marketing propone la captación de la atención del consumidor a través de contenido de calidad, pero bajo el punto de vista de la optimización en cuanto a una correcta difusión y promoción del mismo, consiguiendo no solo el respeto como líder de la industria, sino con el objetivo de incrementar el tráfico cualificado hacia nuestros activos. (p.5)

Además, el documento propone crear la gestión a través del marketing de permisos. El mismo que se construye sobre la base de establecer que el factor esencial de los responsables de la estrategia de marketing es el "de solicitar permiso a los clientes o potenciales clientes a la hora de ejecutar sus acciones." (2011, p. 7)

De esta forma no solo se fomenta un compromiso con los productos y servicios de una forma más cualificada, sino que "(...) se obtiene una mayor capacidad para dirigir la comunicación, de manera directa y personalizada a la audiencia mediante el uso de la información proporcionada por ésta de manera voluntaria (opt-in)". (p. 7) 
Estos factores, son sinónimo del concepto denominado por la autora Wilcock, del marketing de atracción, y especifica al respecto: (...) atracción significa motivar a los consumidores a buscar estas interacciones a través, entre otros, de los contenidos de valor, al contrario que el "marketing de empuje", que requiere de la promoción comercial para acercar el producto a los consumidores a través de los diferentes canales. (p.8)

Por otro lado, es de observar que las empresas continúan realizando acciones de marketing directo, como mailing, campañas en medios tradicionales, telemarketing, y otras actividades tradicionales del marketing. Cuestión que para la autora del PG forma parte de la estrategia online offline, y que además, configuran el escenario de las comunicaciones integradas. Sin embargo, es de importancia, incluir las nuevas bases del marketing para adaptar las estrategias a instancias de convergencia. Puesto que la misma otorga la posibilidad de constituir una red complementaria de actividades con mayor poder de relación. Es de sumar además que, como ya se definió en el PG, el cambio de hábitos de consumo, en gran parte impulsados por las nuevas tecnologías, hace a la afirmación de esta nueva etapa a considerar por los profesionales de la comunicación publicitaria.

Un estudio desarrollado por Drell, el 30 de octubre de 2011, denominado Inbound Marketing vs. Outbound Marketing establece que "el $44 \%$ de los emails directos nunca son abiertos. El $86 \%$ de los consumidores no atienden a los anuncios de televisión. El $84 \%$ de las personas entre 25 y 34 años abandonaron un sitio web a causa de un anuncio irrelevante o intrusivo" (p.9)

Es por este motivo, que el marketing de contenidos aborda el enfoque opuesto. Puesto que proporciona contenido de calidad a los consumidores invitándolos a participar a través del consumo de los mismos. Además bajo un concepto de marketing experiencial es posible obtener mayores niveles de aceptación y de éxito, en términos de tráfico en el sitio web

Es así, como las nuevas formas de relación entre los clientes y las marcas han cambiado. Aumenta la demanda de profesionales capaces de gestionar y crear interacción entre los usuarios y las marcas.

\section{Aplicación del Inbound Marketing}

Una de las primeras empresas en difundir el concepto de Inbound marketing fue Hubspot. Ahora bien, qué es Inbound Marketing y para qué sirve aplicarlo?

El Inbound Marketing es una nueva manera de entender al marketing. Consiste en generar prospectos, y trabajar con ellos para convertirlos en clientes. Se trata de no perder el tiempo y el dinero subcontratando telemarketers, haciendo publicidad masiva, o haciendo "llamadas en frío", o tal vez enviando e-mails que van a parar directo a la 
casilla de basura (Spam). Todas esas técnicas pueden ser conocidas como Outbound marketing

El Marketing de contenidos o Inbound Marketing complementa la forma como los compradores toman las decisiones de compra en la actualidad; como ellos están utilizando el Internet para aprender acerca de los productos y servicios que mejor se adapten a sus necesidades. Los oferentes modernos, se esfuerzan por ofrecer a su público información útil y todo tipo de herramientas para atraer a la gente a su sitio, mientras que también desarrollan maneras eficaces de interactuar y dinamizar las relaciones con las personas en la web. Este tipo de marketing de atracción, como también se le conoce propone herramientas que incluyen blogs, publicación de contenidos, optimización de motores de búsqueda y redes sociales. El documento titulado Inbound Marketing y SEO (Fishkin \& Hogenhaven, 2013) ha puesto de manifiesto los diferentes aspectos de esta novedosa forma de comercialización virtual.

\section{Propuesta de inbound marketing para la cooperativa multiactiva Copservir Ltda}

Elementos integrantes de la propuesta en inbound marketing

\begin{tabular}{|c|c|}
\hline ELEMENTO & META \\
\hline \multirow[b]{2}{*}{$\begin{array}{l}\text { - Buscadores SEO } \\
\text { - Blogosfera } \\
\text { - Redes Sociales } \\
\text { - Whitepaper (informes blancos) }\end{array}$} & 1er Semestre \\
\hline & $\begin{array}{l}\text { - Duplicar el tráfico web } \\
\text { - Duplicar los clientes potenciales del } \\
\text { sitio web } \\
\text { - Incrementar el volumen de visitas a } \\
70 \%\end{array}$ \\
\hline Infografías & 2do Semestre \\
\hline - Webinars & $\begin{array}{l}\text { - Incrementar el tráfico web en un } \\
30 \% \text { más } \\
\text { - } \quad \text { Aumentar el número de clientes } \\
\text { potenciales en un } 30 \% \\
\text { - Incrementar el volumen de } \\
\text { visitas a } 90 \%\end{array}$ \\
\hline
\end{tabular}

\section{Metodología (Paso a Paso Sugerido)}

\section{Atracción de Tráfico Web}

Según Vargas, (2014) uno de los principios básicos del Inbound Marketing consiste en NO salir a buscar prospectos que NO necesitan su producto, sino ponerse al frente de aquellos que ya lo están buscando 
En Colombia el buscador más popular y solicitado es Google, en consecuencia si Copservir Ltda, quiere atraer mayor tráfico web, deberá optimizar su sitio Web. Para este proceso, es necesario utilizar el elemento que se conoce como SEO por sus siglas en inglés: Search Engine Optimization. Para lograr que el sitio Web de la cooperativa (www.copservir.com) Ilegue a los primeros lugares en Google se debería:

- Identificar las palabras clave (keywords) y las frases clave (keyphrases) que utilizarían sus prospectos cuando están buscando su producto o servicio. Piense en todas la opciones posibles ya que cada prospecto puede pensar diferente en el momento de realizar una búsqueda. Esta lista debe ser extensa y exhaustiva. Pensar en posicionar de 200 a 300 keywords no lo debe preocupar. Ejemplo: prevención en salud, belleza, salud física y mental, medicamentos que sirven, la salud a tu alcance, etc. Elemento de inbound marketing asociado: SEO

- Inclusión de las palabras clave: Una vez identificadas las primeras 20 palabras clave o frases clave, es hora de asegurarse incluirlas en su sitio Web. Los lugares donde las debe incluir son: Títulos de página, descripciones de página, encabezados, textos, descripciones alternativas de imágenes, descripciones de videos, artículos de blog y páginas de aterrizaje (landing pages). Elemento de inbound marketing asociado: BLOGOSFERA

- Creación de blogs: El Blog es una herramienta de generación de contenidos muy poderosa que, aprovechada al máximo, puede ayudarle a conseguir muchos prospectos online. Google valora mucho la actualización y creación de nuevas páginas todo el tiempo. Por eso es muy importante que su empresa tenga un blog que publique artículos de interés acerca de los beneficios de sus servicios día a día. Ejemplo: Se puede promover entre los clientes la creación de blogs relacionados a los productos y servicios que ofrece la Cooperativa. Elemento de inbound marketing asociado: BLOGS PERSONALES

- Gestión comercial por medio de las redes sociales: La publicación de contenidos en Internet también debe difundirse a través de las redes sociales. Tener una cuenta en FaceBook, Twitter, Pinterest, Google+, Linkedln y muchas más, le permitirá tener diversos canales para atraer tráfico a su sitio Web.

Elemento de inbound marketing asociado: REDES SOCIALES

\section{Conversión de Visitantes en Leads (Prospectos)}

Vargas, (2014) sostiene que lo más importante de atraer tráfico a su sitio Web es lograr que las visitas duren suficiente tiempo para poder conocer quién lo está visitando y poder hacer un seguimiento a esas visitas.

Para lograr que las visitas a al sitio Web de la cooperativa se conviertan en prospectos se deben crear contenidos de valor que incrementen la permanencia en el sitio y realizar ofertas de contenidos premium que le permitan obtener los datos de sus visitantes a cambio de la descarga gratuita de dichos contenidos.

A continuación se ilustra un ejemplo para esclarecer este proceso: 
- La Cooperativa Multiactiva COPSERVIR Ltda, identifica que en Google hay 2.400 búsquedas mensuales para la palabra clave: "medicamentos libres"

- El equipo de mercadeo de COPSERVIR Ltda escribe un artículo en su Blog que incluye esta palabra clave en los lugares indicados anteriormente.

- Al finalizar el artículo, el equipo de mercadeo de Copservir Ltda, incluye un pequeño banner (call to action) con este anuncio: "Los 10 medicamentos libres más eficaces para la salud - Descargue este "whitepaper" gratis del Blog " $x$ "

- Al hacer clic en el banner, el visitante llega a una página de aterrizaje (Landing page) en donde encuentra una lista con los beneficios que recibirá

- después de leer el Whitepaper y un formulario simple que debe llenar para poder descargarlo.

Elementos de inbound marketing asociados: WHITEPAPERS

\section{Conversión de Prospectos en Clientes}

Una vez sitio Web de la cooperativa (i.e www.copservir.com) comienza a generar prospectos es pertinente comprender que no todos van a estar en listos para convertirse en clientes.

Es aquí donde el equipo de mercadeo debe iniciar un proceso de calificación de prospectos que le permita conocer el estado de preparación y disposición de sus prospectos para convertirse en clientes (el equipo de mercadeo puede tener un prospecto que apenas esté en la fase de investigación y aún no esté listo para comprar y otro que tenga urgencia, ya esté informado y se encuentre listo para convertirse en cliente).

Para poder lograr impactar o "acabar de convencer a este potencial cliente para que pase de una investidura (lead) a un cliente real, se podrá hacer uso de:

- Infografías llamativas de temas de gran interés (como opera el vector del Chikungunya; como el páncreas produce la insulina, como el pulmón se deteriora con la nicotina, etc)

- Webinars: Se pueden proyectar semanalmente o utilizar un software libre en Internet para colgar vídeos relacionados (Por ejemplo, semana del cáncer de mama, video relacionado, manejo posextirpación del seno, etc) Elementos de inbound marketing asociados: INFOGRAFÍAS, VIDEOS ON LINE

\section{Conservación y Profundización de Clientes}

El lograr un grupo de clientes con un volumen de frecuencia si bien es una señal que de que se transita por buen camino, es solo un primer paso para construir una relación de largo plazo que le permita a las empresas crecer de manera rentable y sostenible. Cuando usted logra convertir en cliente a un prospecto de Internet, debe continuar un trabajo de Inbound Marketing para poder conservarlo y posiblemente, profundizarlo con uno o más productos en el futuro. Se recomienda entonces, 
- Mantener un espíritu constante de innovación en los contenidos de la oferta virtual

- Evaluar mediante herramientas de la red, libres o con erogación monetaria incluida, el número de visitantes a las páginas, el conteo de leads nuevos, las tendencias del marketing, etc

- Permitir o dar libertad a los clientes para que ellos sugieran temas, para enriquecer los foros

Elementos de inbound marketing asociados: EMAIL MARKETING

\section{Métricas para medir la efectividad de la propuesta}

Leads (Prospectos) : Por medio del uso de herramientas como Google Analytics se puede registrar la cantidad de prospectos obtenidos de forma diaria semanal y mensual,

Ejemplo:
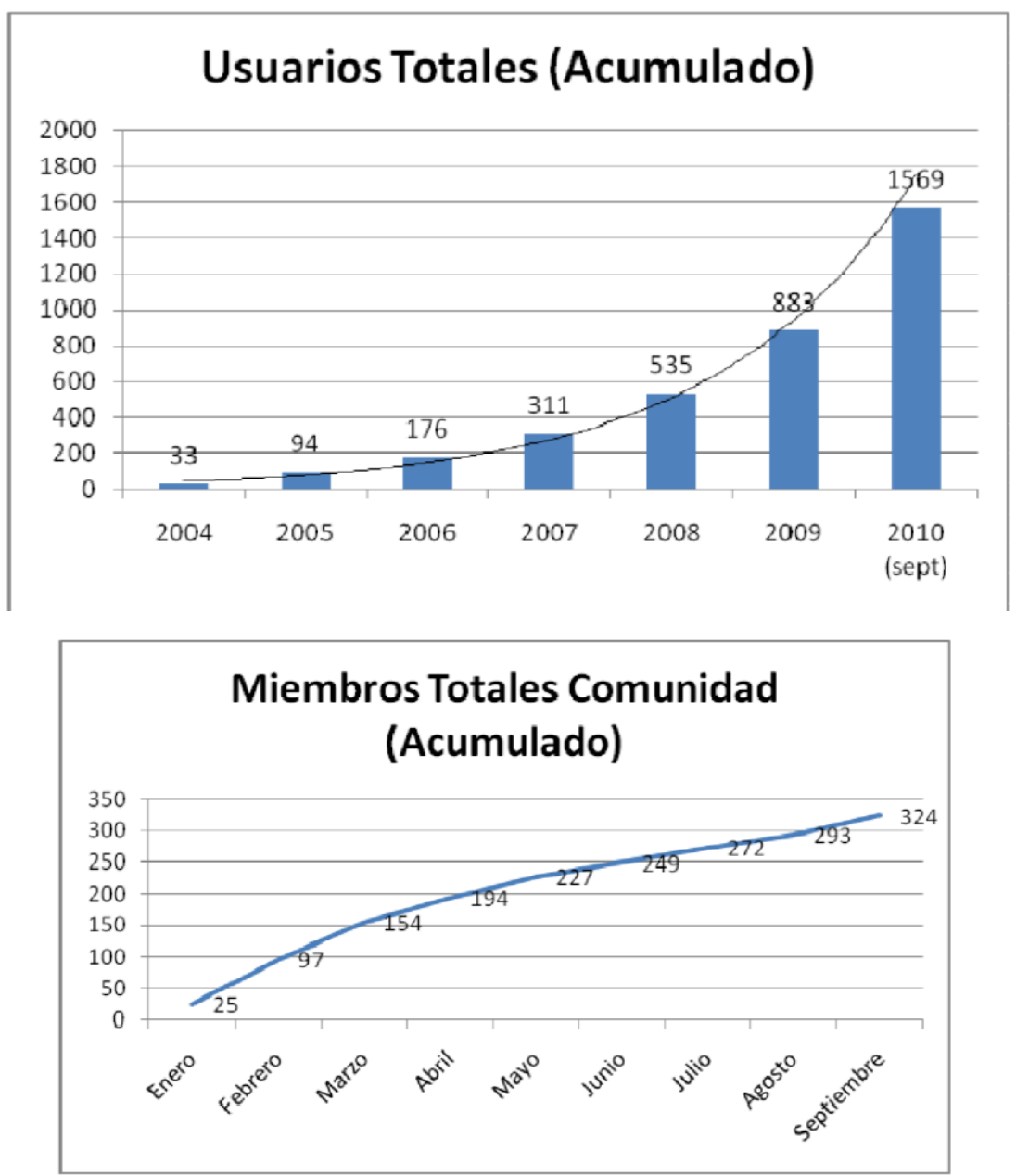

Nuevos Clientes: Son usuarios registrados en algún servicio o que realizan alguna transacción. Si la Cooperativa aun no lleva un análisis de estos usuarios puede ser implementado con variables definidas en Google Analytics.

Transacciones: Veces al año, o al mes, en las que compra un cliente en los portales, o a domicilio presencial, etc 
Ejemplo:

\section{Métricas del sitio Web}

- Tráfico del Sitio Web: Visitas y usuarios del sitio web

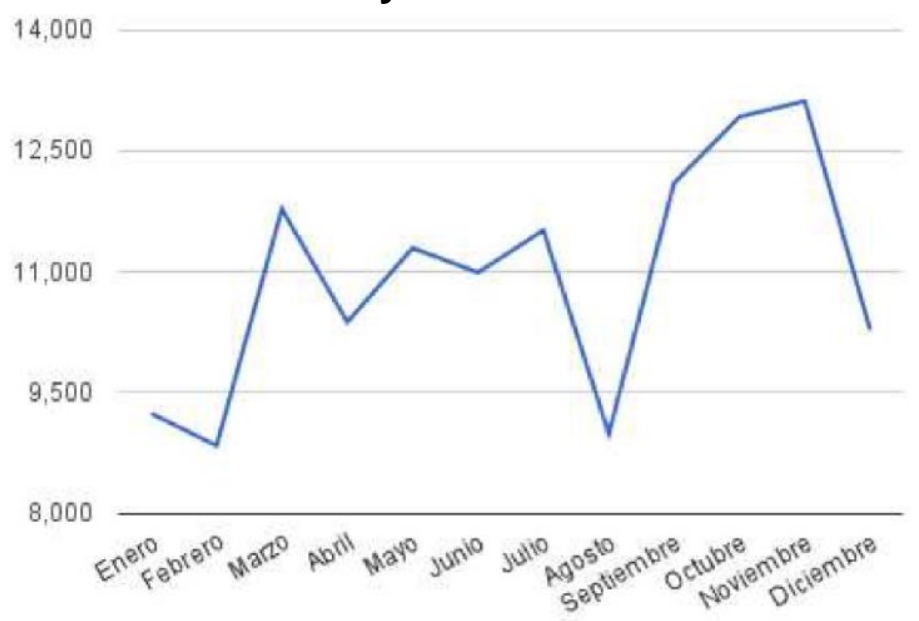

- Tiempo promedio en el sitio: Interacción de los visitantes en el sitio web

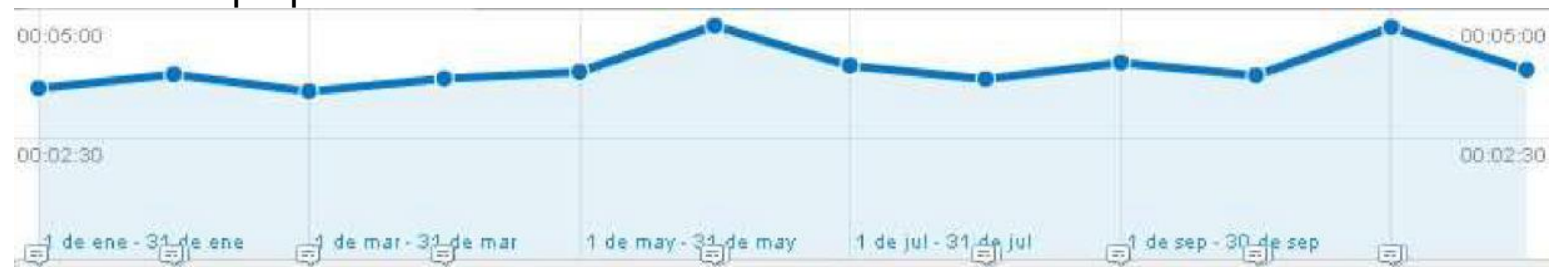

\section{Métrica para SEO}

Esta métrica hace la medición del origen de las visitas al sitio web. En este ejemplo se puede visualizar el incremento del tráfico desde otros sitios, lo que indica que se ha incrementado las visitas desde otros sitios y desde campañas realizadas. 


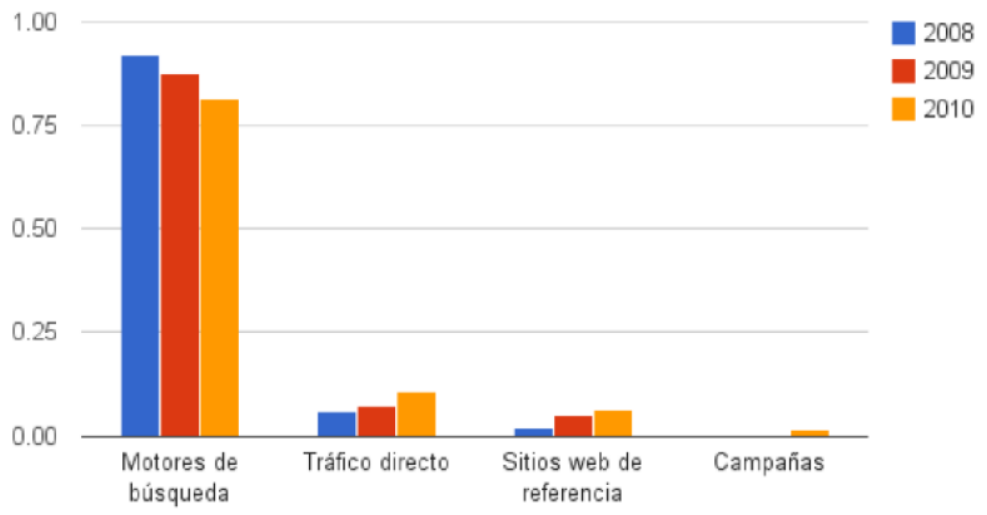

\section{Métrica para blogs}

Suscripciones: Esta métrica indica el número de personas que se han registrado a los diferentes blogs de la Cooperativa, para esto se puede utilizar la herramienta feedburner de Google.

Ejemplo:

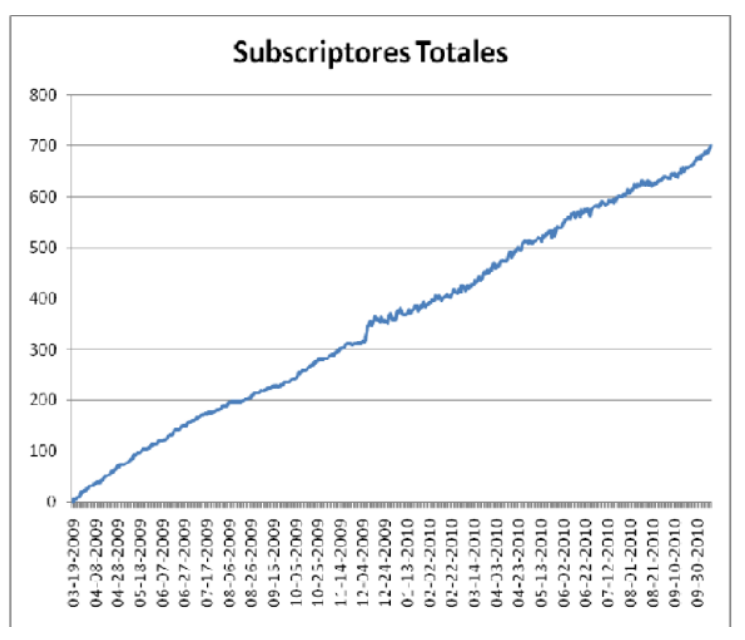

\section{Métrica para redes sociales}

Twitter: Mediante la herramienta Twitter Counter se puede medir la cantidad de Seguidores, Tweets y Cuentas Seguidas.

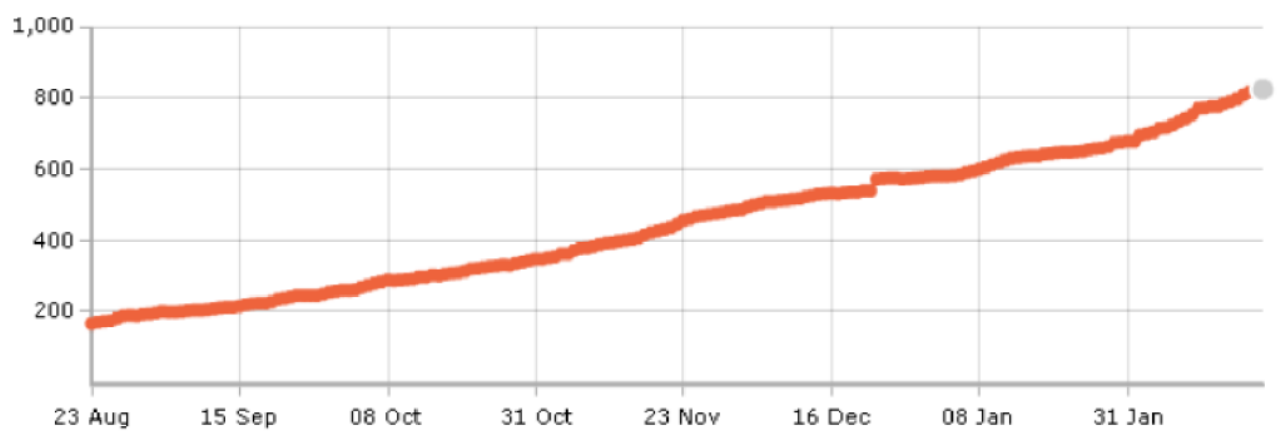


Facebook: Si la Cooperativa cuenta con una Fan Page, o si la crea el equipo de marketing se puede ver las métricas: Páginas Vistas y Fans.

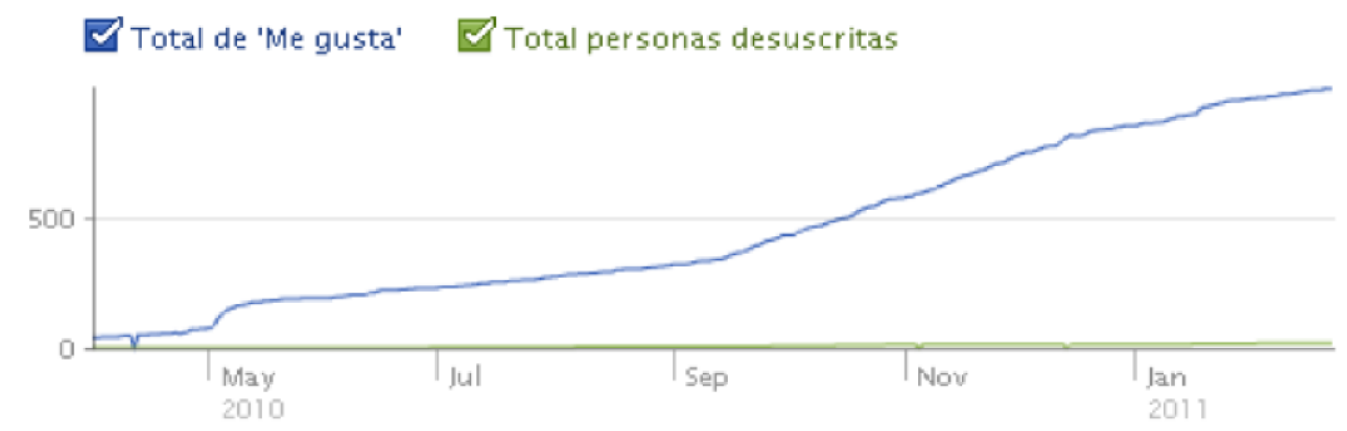

Youtube: En este canal de videos se puede medir las veces que se ha visto un video y los suscriptores que ha tenido.

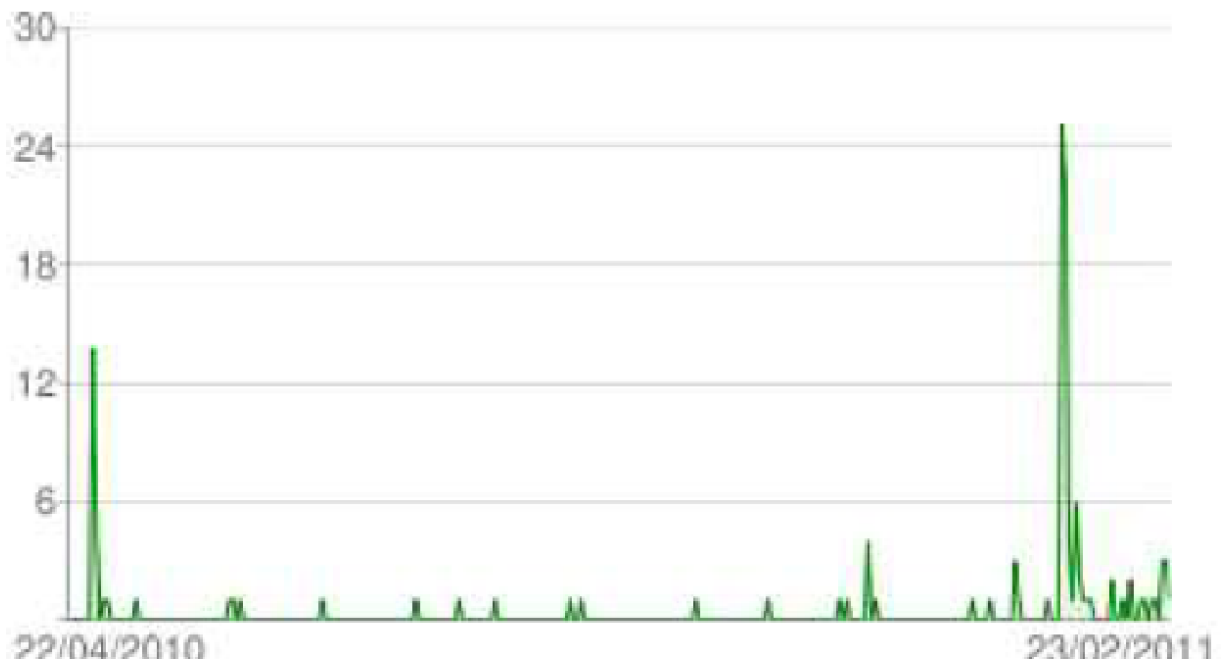

Linkedin: En la sección Analytics de Linkedln se puede medir las Páginas vistas y los visitantes de dicha página, como se puede apreciar en este ejemplo

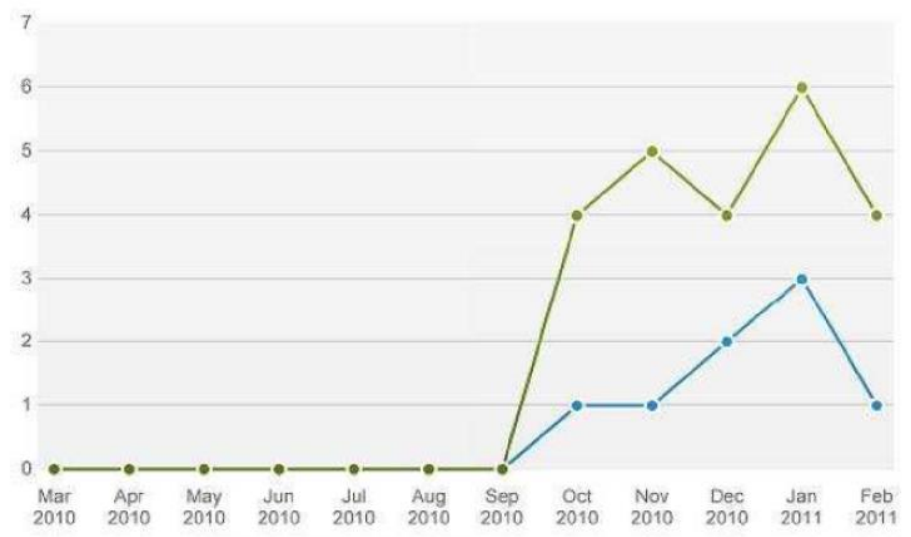




\section{Resultados y discusión}

A favor de Copservir y su no utilización del Inbound Marketing obedece estrictamente a su desconocimiento, porque este es un modelo relativamente nuevo. Lo que resulta no tan justificable, es que teniendo ya un proceso iniciado de virtualización comercial de la compañía, no se haya retroalimentado objetivamente, respecto al aprovechamiento idóneo de este portal.

El hecho o la omisión para contextualizar la contingencia, ha conllevado a que la organización crea que el crecimiento de clientes es lento, a pesar de estar "en línea" para la comunidad, lo que amerita que la cooperativa reformules sus objetivos corporativos, para que pueda obtener un mejor desempeño en el mercado.

\section{Conclusiones}

La investigación que se llevó a cabo con la cooperativa multiactiva Copservir Ltda, sobre el estudio sobre estrategias de Inbound Marketing ha conducido a la conclusión de que en esta organización el tema de la gestión comercial en lo inherente a su oferta virtual resultaba insuficiente, si bien esta organización goza de una oferta abierta y de con un gran cobertura, se puede considerar limitada.

Según la investigación realizada, la organización si bien se ha mostrado solicita al uso intensivo de la virtualidad, aún tiene mucho potencial para mejorar y optimizar, un adagio muy preciso dice que...no basta estar en el camino correcto, hay que caminar en él...esto pareciera justo para describir la gestión comercial que ha tenido este importante referente de oferta farmacéutica, no basta entonces tener presencia en la red, sino que la misma debe de ser eficiente.

La ventaja, no obstante para esta organización, una vez se ha terminado este ejercicio investigativo, es que la cooperativa como tal es uno de los referentes más representativos del sector farmacéutico nacional, lo que potencializa la posibilidad de mejorar aún más su posicionamiento, hoy en día, es la segunda empresa más importante de este sector.

Con esta propuesta del uso de las herramientas y elementos del Inbound Marketing, se puede lograr este propósito, y quizás sea la conclusión más relevante que deja esta investigación, que la cooperativa tiene todas las posibilidades de éxito.

Literatura citada

Fishkin, R., \& Hogenhaven, T. (2013). Inbound marketing and SEO: Insights from the Moz Blog. Wiley.

Simpson, P., \& Jevons, C. (2009). Internet-enabled International Marketing: A Small Business. 
Vargas, S. (2014). Por qué debe implementar una estrategia de inbound marketing si quiere crecer sus ventas. Bogotá: Giraffe.

Wilcok, M. (2011). Marketing de Contenidos. Crear para convertir. España. 